\title{
ON GENERALISED CONVEX MATHEMATICAL PROGRAMMING
}

\author{
V. JEYAKUMAR ${ }^{1}$ and B. MOND ${ }^{2}$
}

(Received 22 February 1991; revised 21 March 1991)

\begin{abstract}
The sufficient optimality conditions and duality results have recently been given for the following generalised convex programming problem:

$$
\text { Minimise } f(x) \text {, subject to } g(x) \leq 0, x \in X_{0} \subset \mathbf{R}^{n} \text {, }
$$

where the functions $f$ and $g$ satisfy

$$
x, a \in X_{0} \Rightarrow\left\{\begin{array}{l}
f(x)-f(a)-f^{\prime}(a) \eta(x, a) \geq 0 \\
g(x)-g(a)-g^{\prime}(a) \eta(x, a) \geq 0,
\end{array}\right.
$$

for some $\eta: X_{0} \times X_{0} \rightarrow \mathbb{R}^{n}$.

It is shown here that a relaxation defining the above generalised convexity leads to a new class of multi-objective problems which preserves the sufficient optimality and duality results in the scalar case, and avoids the major difficulty of verifying that the inequality holds for the same function $\eta(.,$.$) . Further, this relaxation allows$ one to treat certain nonlinear multi-objective fractional programming problems and some other classes of nonlinear (composite) problems as special cases.
\end{abstract}

\section{Introduction}

Consider the constrained multi-objective optimisation problem

(VP) V-Minimise $\left(f_{1}(x), \ldots, f_{p}(x)\right)$ subject to $g(x) \leq 0$, where $f_{i}: X_{0} \rightarrow \mathbb{R}$ and $g: X_{0} \rightarrow \mathbb{R}^{m}$ are differentiable functions and $X_{0}$ is an open set in $\mathbb{R}^{n}$. Note here that the symbol "V-Minimise" stands for vector minimisation. This is the problem of finding the set of weak minimum

\footnotetext{
${ }^{1}$ School of Mathematics, University of New South Wales, Kensington, NSW, Australia 2033.

${ }^{2}$ School of Mathematics and Information Sciences, La Trobe University, Vic. Australia 3083.

(C) Copyright Australian Mathematical Society 1992, Serial-fee code 0334-2700/92
} 
[13] for points (VP). When $p=1$, the problem (VP) reduces to a scalar optimisation problem and it is denoted by $(\mathrm{P})$. Convexity of the scalar problem $(P)$ is characterised by the inequalities

$$
\begin{aligned}
& f_{1}(x)-f_{1}(a)-f_{1}{ }^{\prime}(a) \quad(x-a) \geq 0 \\
& g(x)-g(a)-g^{\prime}(a)(x-a) \geq 0, \quad \forall x, a \in X_{0} .
\end{aligned}
$$

Hanson [9] observed that the functional form $(x-a)$ here plays no role in establishing the following two well-known properties in scalar convex programming:

(S) Every feasible Kuhn-Tucker point is a global minimum .

(W) Weak duality holds between (P) and its associated dual problem.

Having this in mind, Hanson [9] considered problem (P) for which there exists a function $\eta: X_{0} \times X_{0} \rightarrow \mathbb{R}^{n}$ such that

$$
\begin{aligned}
& f_{1}(x)-f_{1}(a)-f_{1}(a)^{\prime} \quad \eta(x, a) \geq 0 \\
& g(x)-g(a)-g^{\prime}(a) \eta(x, a) \geq 0
\end{aligned} \quad \forall x, a \in X_{0},
$$

and showed that such problems (known now as invex problems $[4,5,8]$ ) also possess properties (S) and (W). Since then, various generalisations of conditions (I) to multi-objective problems and many properties of functions that satisfy (I) have been established in the literature, e.g. $[1,5,8,10]$. However, the major difficulty is that the invex problems require the same function $\eta(x, a)$ for the objective function and the constraints. This requirement turns out to be a severe restriction in applications. Because of this restriction, pseudolinear multi-objective problems [3] and certain nonlinear multiobjective fractional programming problems require separate treatment as far as optimality and duality properties are concerned.

In this paper we show how this situation can be improved and how the properties (S) and (W) can be extended to hold for generalised invex multiobjective problems and certain multi-objective fractional problems. To this end, we modify the condition (I) in the next section as follows.

\section{New classes of generalised convex vector functions}

A vector function $f: X_{0} \rightarrow \mathbb{R}^{p}$ is said to be $V$-invex if there exist functions $\mu: X_{0} \times X_{0} \rightarrow \mathbb{R}^{p}$ and $\alpha_{i}: X_{0} \times X_{0} \rightarrow \mathbb{R}_{+} \backslash\{0\}$ such that for each $x, a \in X_{0}$, and for $i=1,2, \ldots, p$,

$$
f_{i}(x)-f_{i}(a)-\alpha_{i}(x, a) f_{i}^{\prime}(a) \eta(x, a) \geq 0 .
$$


When $p=1$, the definition of $\mathrm{V}$-invexity reduces to the notion of invexity in the sense of Hanson [9] with $\eta(x, a) \equiv \alpha_{1}(x, a) \eta(x, a)$. When $p=1$ and $\eta(x, a) \equiv x-a$, the condition reduces to strong pseudo-convexity condition (see $[11,2])$. The problem (VP) is said to be $\mathrm{V}$-invex if the vector function $\left(f_{1}, \ldots, f_{p}, g_{1}, \ldots, g_{m}\right)$ is V-invex. Equivalently, V-invexity of (VP) means that there exist functions $\mu: X_{0} \times X_{0} \rightarrow \mathbb{R}^{n}$ and $\alpha_{i}, \beta_{j}: X_{0} \times$ $X_{0} \rightarrow \mathbb{R}_{+} \backslash\{0\}, i=1,2, \ldots, p, j=1,2, \ldots, m$ such that

(VI) $\quad x, a \in X_{0} \Rightarrow\left\{\begin{array}{l}f_{i}(x)-f_{i}(a)-\alpha_{i}(x, a) f_{i}^{\prime}(a) \eta(x, a) \geq 0 \\ g_{j}(x)-g_{j}(a)-\beta_{j}(x, a) g_{j}^{\prime}(a) \eta(x, a) \geq 0 .\end{array}\right.$

The invex [resp. convex] case corresponds of course to $\alpha_{i}(x, a)=1$, for $i=1,2, \ldots, p$, and $\beta_{j}(x, a)=1$, for $j=1,2, \ldots, m$ [resp. $\eta(x, a) \equiv$ $x-a$, and $\alpha_{i}(x, a)=1=\beta_{j}(x, a)$, for $\left.i=1,2, \ldots, p, j=1,2, \ldots, m\right]$. When $\eta(x, a) \equiv x-a$ and $p=1$, the scalar problem (P) becomes the strongly pseudo-convex programming problem [2]. Note also that linear fractional multi-projective programs and pseudolinear multi-objective programs [3] are V-invex problems. Moreover, invex multi-objective problems are necessarily V-invex, but not conversely. For a simple example, consider

$$
\min _{x_{1}, x_{2} \in \mathbf{R}}\left(\frac{x_{1}^{2}}{x_{2}}, \frac{x_{2}}{x_{1}}\right) \text { subject to } 1-x_{1} \leq 0,1-x_{2} \leq 0 \text {. }
$$

Then, it is easy to see that this problem satisfies the condition (VI) with $\alpha_{1}(\underset{\sim}{x}, \underset{\sim}{a})=a_{2} / x_{2}, \alpha_{2}(\underset{\sim}{x} \underset{\sim}{a})=a_{1} / x_{1}, \beta_{1}(\underset{\sim}{x}, \underset{\sim}{a})=1$, for $=1,2$, and $\eta(x, a)=x-a$; but clearly, the problem does not satisfy the invexity condition with the same $\eta$. It is also worth noting that the functions involved in (VP) are invex, but (VP) is not necessarily invex.

It is known that invex problems can be constructed from convex problems by certain nonlinear coordinate transformations, see [4, 5]. In the following example, we see that $\mathrm{V}$-invex functions (problems) can be formed from certain nonconvex functions (problems) [in particular, from convex-concave or linear fractional functions (problems)] via coordinate transformations.

EXAMple 2.1. Consider the function $h: \mathbb{R}^{n} \rightarrow \mathbb{R}^{p}$ defined by

$$
h(x)=\left(f_{1}(\phi(x)), \ldots, f_{p}(\phi(x))\right),
$$

where $f_{i}: \mathbb{R}^{n} \rightarrow \mathbb{R}, i=1,2, \ldots, p$, are strongly pseudo-convex functions with real positive functions $\alpha_{i}(x, a), \phi: \mathbb{R}^{n} \rightarrow \mathbb{R}^{n}$ is surjective with $\phi^{\prime}(a)$ onto for each $a \in \mathbb{R}^{n}$. Then, the function $h$ is $\mathrm{V}$-invex. To see this, let $x$, $a \in \mathbb{R}^{n}, u=\phi(x), v=\phi(a)$. Then, by strong pseudo-convexity,

$$
\begin{aligned}
f_{i}(\phi(x))-f_{i}(\phi(a)) & =f_{i}(u)-f_{i}(v) \\
& \geq \alpha_{i}(u, v) f_{i}^{\prime}(v)(u-v) .
\end{aligned}
$$


Since $\phi^{\prime}(a)$ is onto, $u-v=\phi^{\prime}(a) \eta(x, a)$ is solvable for some $\eta(x, a) \in \mathbb{R}^{n}$. Hence,

$$
\begin{aligned}
f_{i}(\phi(x))-f_{i}(\phi(a)) & \geq \alpha_{i}(u, v) f_{i}^{\prime}(v) \phi^{\prime}(a) \eta(x, a) \\
& =\bar{\alpha}_{i}(x, a)\left(f_{i} \circ \phi\right)^{\prime}(a) \eta(x, a),
\end{aligned}
$$

where $\bar{\alpha}_{i}(x, a)=\alpha_{i}(\phi(x), \phi(a))>0$.

EXAMPLE 2.2. Consider the composite vector function $h(x)=\left(f_{1}\left(F_{1}(x)\right)\right.$, $\left.\ldots, f_{p}\left(F_{p}(x)\right)\right)$, where for each $i=1,2, \ldots, p, F_{i}: X_{0} \rightarrow \mathbb{R}$ is continuously differentiable and pseudolinear with the positive proportional function $\alpha_{i}(.,$.$) , and f_{i}: \mathbb{R} \rightarrow \mathbb{R}$ is convex. Then, $h$ is V-invex with $\eta(x, a)=x-a$. This follows from the following convex inequality and pseudolinear equality conditions:

$$
\begin{aligned}
f_{i}\left(F_{i}(x)\right)-f_{i}\left(F_{i}(a)\right) & \geq f_{i}^{\prime}\left(F_{i}(a)\right)\left(F_{i}(x)-F_{i}(a)\right) \\
& =f_{i}^{\prime}\left(F_{i}(a)\right) \alpha_{i}(x, a) F_{i}^{\prime}(a)(x-a) \\
& =\alpha_{i}(x, a)\left(f_{i} \circ F_{i}\right)^{\prime}(a)(x-a) .
\end{aligned}
$$

For a simple numerical example of a composite vector function, consider $h\left(x_{1}, x_{2}\right)=\left(e^{x_{1} / x_{2}}, \frac{x_{1}-x_{2}}{x_{1}+x_{2}}\right)$, where $X_{0}=\left\{\left(x_{1}, x_{2}\right) \in \mathbb{R}^{2} \mid x_{1} \geq 1, x_{2} \geq 1\right\}$.

EXAMPLE 2.3. Consider the function

$$
p(x)=\left(f_{1}\left(\left(h_{1} \circ \psi\right)(x)\right), \ldots, f_{p}\left(\left(h_{p} \circ \psi\right)(x)\right)\right),
$$

where each $h_{i}$ is pseudolinear on $\mathbb{R}^{n}$ with proportional functions $\alpha_{i}(x, a)$, $\psi$ is a differentiable mapping from $\mathbb{R}^{n}$ onto $\mathbb{R}^{n}$ such that $\psi^{\prime}(a)$ is surjective for each $a \in \mathbb{R}^{n}$, and $f_{i}: \mathbb{R} \rightarrow \mathbb{R}$ convex for each $i$. Then $p$ is $\mathrm{V}$-invex. To see this, let. $u=\psi(x), v=\psi(a)$. Then, by the pseudolinearity condition, we get

$$
\begin{aligned}
h_{i}(\psi(x))-h_{i}(\psi(a)) & =h_{i}(u)-h_{i}(v) \\
& =\alpha_{i}(u, v) h_{i}^{\prime}(v)(u-v) .
\end{aligned}
$$

Since $\psi^{\prime}(a)$ is onto, $u-v=\psi^{\prime}(a) \eta(x, a)$ is solvable for some $\eta(x, a) \in \mathbb{R}^{n}$. Hence,

$$
\begin{aligned}
h_{i}(\psi(x))-h_{i}(\psi(a)) & =\alpha_{i}(u, v) h_{i}^{\prime}(v) \psi^{\prime}(a) \eta(x, a) \\
& =\bar{\alpha}_{i}(x, a)\left(h_{i} \circ \psi\right)^{\prime}(a) \eta(x, a),
\end{aligned}
$$

where $\bar{\alpha}_{i}(x, a)=\alpha_{i}(\psi(x), \psi(a))>0$. Hence,

$$
\begin{aligned}
f_{i}\left(\left(h_{i} \circ \psi\right)(x)\right)-f_{i}\left(\left(h_{i} \circ \psi\right)(a)\right) & \geq f_{i}^{\prime}\left(\left(h_{i} \circ \psi\right)(a)\right)\left(\left(h_{i} \circ \psi\right)(x)-\left(h_{i} \circ \psi\right)(a)\right) \\
& =f_{i}^{\prime}\left(\left(h_{i} \circ \psi\right)(a)\right) \bar{\alpha}_{i}(x, a)\left(h_{i} \circ \psi\right)^{\prime}(a) \eta(x, a) .
\end{aligned}
$$


We now show that the $\mathrm{V}$-invexity is preserved under a smooth convex transformation.

Proposition 2.1. Let $\psi: \mathbb{R} \rightarrow \mathbb{R}$ be differentiable and convex with positive derivative everywhere; let $h: X_{0} \rightarrow \mathbb{R}^{p}$ be V-invex. Then, the function

$$
h_{\psi}(x)=\left(\psi\left(h_{1}(x)\right), \ldots, \psi\left(h_{p}(x)\right), \quad x \in X_{0}\right.
$$

is V-invex.

Proof. Let $x, a \in X_{0}$. Then, from the monotonicity of $\psi$ and V-invexity of $h$, we get

$$
\begin{aligned}
\psi\left(h_{i}(x)\right) & \geq \psi\left(h_{i}(a)+\alpha_{i}(x, a) h_{i}^{\prime}(a) \eta(x, a)\right) \\
& \geq \psi\left(h_{i}(a)\right)+\psi^{\prime}\left(h_{i}(a)\right) \alpha_{i}(x, a) h^{\prime}(a) \eta(x, a) \\
& =\psi\left(h_{i}(a)\right)+\alpha_{i}(x, a)\left(\psi \circ h_{i}\right)^{\prime}(a) \eta(x, a) .
\end{aligned}
$$

Thus, $h_{\psi}(x)$ is V-invex.

The notions of pseudo-invexity and quasi-invexity for a scalar function can now be extended to a vector function. A vector function $f: X_{0} \rightarrow \mathbb{R}^{p}$ is said to be V-pseudo-invex if there exist functions $\mu: X_{0} \times X_{0} \rightarrow \mathbb{R}^{p}$ and $\beta_{i}: X_{0} \times X_{0} \rightarrow \mathbb{R}_{+} \backslash\{0\}$ such that for each $x, a \in X_{0}$,

$$
\sum_{i=1}^{p} f_{i}^{\prime}(a) \eta(x, a) \geq 0 \Rightarrow \sum_{i=1}^{p} \beta_{i}(x, a) f_{i}(x) \geq \sum_{i=1}^{p} \beta_{i}(x, a) f_{i}(a) .
$$

The vector function $f$ is said to be $\mathbf{V}$-quasi-invex if there exist functions $\mu: X_{0} \times X_{0} \rightarrow \mathbb{R}^{p}$ and $\gamma_{i}: X_{0} \times X_{0} \rightarrow \mathbb{R}_{+} \backslash\{0\}$ such that for each $x, a \in X_{0}$,

$$
\sum_{i=1}^{p} \gamma_{i}(x, a) f_{i}(x) \leq \sum_{i=1}^{p} \gamma_{i}(x, a) f_{i}(a) \Rightarrow \sum_{i=1}^{p} f_{i}^{\prime}(a) \eta(x, a) \leq 0 .
$$

It is apparent from the condition (VI) that every V-invex function is Vpseudo-invex and $V$-quasi-invex. Recall that a point $a \in \mathbb{R}^{n}$ is said to be a (global) weak minimum of a vector function $f: \mathbb{R}^{n} \rightarrow \mathbb{R}^{p}$ if there exists no $x \in \mathbb{R}^{n}$ for which $f_{i}(x)<f_{i}(a), i=1,2, \ldots, p$.

Proposition 2.2. Let $f: \mathbb{R}^{n} \rightarrow \mathbb{R}^{p}$ be V-invex. Then $a \in \mathbb{R}^{n}$ is a (global) weak minimum of $f$ if and only if there exists $0 \neq \tau \in \mathbb{R}^{p}, \tau_{i} \geq 0$, $\sum_{i=1}^{p} \tau_{i} f_{i}^{\prime}(a)=0$.

Proof. $(\Rightarrow)$ Suppose that $a$ is weak minimum for $f$. Then the following linear system

$$
x \in \mathbb{R}^{n}, \quad f_{i}^{\prime}(a) x<0, \quad i=1,2, \ldots, p,
$$


is inconsistent. Hence, the conclusion follows from the Gordan Alternative Theorem [7].

$(\Leftarrow)$ Assume that $\sum_{i=1}^{p} \tau_{i} f_{i}^{\prime}(a)=0$, for some $0 \neq \tau \in \mathbb{R}^{p}, \tau_{i} \geq 0$. Suppose that $a$ is not a weak minimum for $f$. Then there exists $x_{0} \in \mathbb{R}^{n}$ such that $f_{i}\left(x_{0}\right)<f_{i}(a), i=1,2, \ldots, p$. Since $f$ is $\mathrm{V}$-invex, there exist $\alpha_{i}\left(x_{0}, a\right)>0, i=1,2, \ldots, p$, and $\eta\left(x_{0}, a\right) \in \mathbb{R}^{n}$ such that

$$
\frac{1}{\alpha_{i}\left(x_{0}, a\right)}\left(f_{i}\left(x_{0}\right)-f_{i}(a)\right) \geq f_{i}^{\prime}(a) \eta\left(x_{0}, a\right) \text {. }
$$

So, $\sum_{i=1}^{p}\left(\tau_{i} / \alpha_{i}\left(x_{0}, a\right)\right)\left(f_{i}\left(x_{0}-f_{i}(a)\right)<0\right.$, and hence $\sum_{i=1}^{p} \tau_{i} f_{i}^{\prime}(a) \eta\left(x_{0}, a\right)<$ 0 . This is a contradiction.

Remark 2.1. From Proposition 2.2 we see that for a V-invex vector function every critical point (i.e. $\left.f_{i}^{\prime}(a)=0, i=1,2, \ldots, p\right)$ is a global weak minimum.

\section{Sufficiency and duality}

In this section we extend the properties (S) and (W) for the multi-objective V-invex problem.

THEOREM 3.1. Consider the multi-objective problem (VP). Suppose that the Lagrange multiplier conditions that $\sum_{i=1}^{p} \tau_{i} f_{i}^{\prime}(a)+\sum_{j=1}^{m} \lambda_{j} g_{j}^{\prime}(a)=0$, $\lambda_{j} g_{j}(a)=0, \tau \in \mathbb{R}^{p}, \tau \neq 0, \tau \geq 0, \lambda \in \mathbb{R}^{m}, \lambda \geq 0$, hold at a feasible point $a \in X_{0}$. If $\left(\tau_{1} f_{1}, \ldots, \tau_{p} f_{p}\right)$ is $V$-pseudo-invex with respect to $\mu$ and $\left(\lambda_{1} g_{1}, \ldots, \lambda_{m} g_{m}\right)$ is $V$-quasi-invex with respect to $\mu$ then $a$ is a global weak minimum point for (VP).

Proof. Suppose that $a$ is not a global minimum point. Then there exists feasible $x_{0} \in X_{0}$ such that $f_{i}\left(x_{0}\right)<f_{i}(a), i=1,2, \ldots, p$. So, $\sum_{i=1}^{p} \beta_{i}\left(x_{0}, a\right) \tau_{i} f_{i}\left(x_{0}\right)<\sum_{i=1}^{p} \beta_{i}\left(x_{0}, a\right) \tau_{i} f_{i}(a)$. Now by the V-pseudoinvexity condition, we get $\sum_{i=1}^{p} \tau_{i} f_{i}^{\prime}(a) \mu\left(x_{0}, a\right)<0$. Since the Lagrangian conditions hold at $a$,

$$
\sum_{j=1}^{m} \lambda_{j} g_{j}^{\prime}(a) \mu\left(x_{0}, a\right)>0 .
$$

From the V-quasi-invexity condition, we get

$$
\sum_{j=1}^{m} \gamma_{j}\left(x_{0}, a\right) \lambda_{j} g_{j}\left(x_{0}\right)>\sum_{j=1}^{m} \gamma_{j}\left(x_{0}, a\right) \lambda_{j} g_{j}(a) \text {. }
$$


This is a contradiction, since

$$
\lambda_{j} g_{j}\left(x_{0}\right) \leq 0, \quad \lambda_{j} g_{j}(a)=0,
$$

and

$$
\gamma_{j}\left(x_{0}, a\right)>0, \quad \text { for } j=1,2, \ldots, m \text {. }
$$

Note that Theorem 3.1 holds if, in particular, the problem (VP) is V-invex. In the scalar case Theorem 3.1 extends the corresponding result of Hanson [9].

For the problem (VP), consider a corresponding dual problem

$$
\begin{aligned}
& \text { V-Maximise }\left(f_{1}(\xi), \ldots, f_{p}(\xi)\right) \\
& \text { (VD) } \quad \text { subject to } \sum_{i=1}^{p} \tau_{i} f_{i}^{\prime}(\xi)+\sum_{j=1}^{m} \lambda_{j} g_{j}^{\prime}(\xi)=0, \\
& \lambda \geq 0, \tau \geq 0, \tau e=1, \quad \lambda_{j} g_{j}(\xi) \geq 0, j=1,2, \ldots, m,
\end{aligned}
$$

where $e=(1, \ldots, 1)$. Note that the Lagrangian conditions in Theorem 3.1 hold for (VP) at a weak minimum point $a$ under a constraint qualification and they can equivalently be written as $\sum_{i=1}^{p} \tau_{i} f_{i}^{\prime}(a)+\sum_{j=1}^{m} \lambda_{j} g_{j}^{\prime}(a)=0, \tau \geq$ $0, \tau e=1, \lambda \geq 0, \lambda_{j} g_{j}(a)=0, j=1,2, \ldots, m$, (see [13]). We now see that appropriate duality results between (VP) and (VD) hold.

Theorem 3.2 (Weak Duality). Consider the multi-objective problems (VP) and (VD). Let $x$ be feasible for (VP) and let $(\xi, \tau, \lambda)$ be feasible for (VD). If $\left(\tau_{1}, f_{1}, \ldots, \tau_{p} f_{p}\right)$ is $V$-pseudo-invex and $\left(\lambda_{1} g_{1}, \ldots, \lambda_{m} g_{m}\right)$ is $V$. quasi-invex with respect to the same $\mu$, then

$$
\left(f_{1}(x), \ldots, f_{p}(x)\right)^{T}-\left(f_{1}(\xi), \ldots, f_{p}(\xi)\right)^{T} \notin-\text { int } \mathbb{R}_{+}^{p} .
$$

Proof. From the feasibility conditions,

$$
\lambda_{j} g_{j}(x)-\lambda_{j} g_{j}(\xi) \leq 0, \quad \text { for each } j=1,2, \ldots, m .
$$

Since $\gamma_{j}(x, \xi)$ is positive,

$$
\sum_{i=1}^{m} \gamma_{j}(x, \xi) \lambda_{j}\left[g_{j}(x)-g_{j}(\xi)\right] \leq 0 .
$$

Hence, $\sum_{j=1}^{m} \lambda_{j} g_{j}^{\prime}(\xi) \mu(x, \xi) \leq 0$, and so, $\sum_{i=1}^{p} \tau_{i} f_{i}^{\prime}(\xi) \mu(x, \xi) \geq 0$. The conclusion now follows from the V-pseudo-invexity condition since $\tau e=1$ and $\beta_{i}(x, \xi)>0$. 
THEOREM 3.3 (Strong Duality). Assume that $a$ is a weak minimum of (VP) and that a suitable constraint qualification is satisfied at $a$. Then there exist $(\tau, \lambda)$ such that $(a, \tau, \lambda)$ is feasible for (VD) and the objective functions of (VP) and (VD) are equal at these points. If, also, for all feasible $(\xi, \tau, \lambda)$, $\left(\tau_{1} f_{1}, \ldots, \tau_{p} f_{p}\right)$ is V-pseudo-invex and $\left(\lambda_{1} g_{1}, \ldots, \lambda_{m} g_{m}\right)$ is V-quasi-invex, then $(a, \tau, \lambda)$ is weak maximum for (VD).

Proof. Since $a$ is a weak minimum for $(\mathrm{P})$ and a constraint qualification is satisfied at $a$, from the Lagrangian conditions (see Theorem 3.1), there exists $(\tau, \lambda)$ such that $(a, \tau, \lambda)$ is feasible for (VD). Clearly the values of (VP) and (VD) are equal at $a$, since the objective functions for both problems are the same. By the generalised V-invexity hypotheses, weak duality holds; hence if $(a, \tau, \lambda)$ is not a weak optimum for (VD), there must exist $\left(\xi, \tau^{*}, \lambda^{*}\right)$ feasible for (VD), $\xi \neq a$, such that

$$
\left(f_{1}(\xi), \ldots, f_{p}(\xi)\right)^{T}-\left(f_{1}(a), \ldots, f_{p}(a)\right)^{T} \in \text { int } \mathbb{R}_{+}^{p},
$$

contradicting weak duality.

\section{Nonconvex multi-objective fractional programming}

In this section we apply the results of the previous section to study fractional nonconvex multi-objective problems.

Consider the fractional programming problem

$$
\begin{gathered}
\text { V-Minimise }\left(p_{1}(x) / q_{1}(x), \ldots, p_{r}(x) / q_{r}(x)\right) \\
\text { subject to } x \in X_{0}, g(x) \leq 0 .
\end{gathered}
$$

where $p_{i}: X_{0} \rightarrow \mathbb{R}, q_{i}: X_{0} \rightarrow \mathbb{R}$ and $g: X_{0} \rightarrow \mathbb{R}^{m}$. We assume that $p_{i}(x) \geq$ 0 , for each $x$ on the feasible set $\Delta=\left\{x \in X_{0} \mid g(x) \leq 0\right\}, q_{i}(x)>0$, for each $x \in \Delta$. The problem (FI) is said to be a V-invex fractional problem if the functions $p, q$ and $g$ satisfy

$$
x, a \in \Delta \Rightarrow\left\{\begin{array}{l}
p_{i}(x)-p_{i}(a) \geq \gamma_{i}(x, a) p_{i}^{\prime}(a) \eta(x, a) \\
q_{i}(x)-q_{i}(a) \leq \gamma_{i}(x, a) q_{i}^{\prime}(a) \eta(x, a) \\
g_{j}(x)-g_{j}(a) \geq \beta_{j}(x, a) g_{j}^{\prime}(a) \eta(x, a),
\end{array}\right.
$$

with $\mu: X_{0} \times X_{0} \rightarrow \mathbb{R}^{n}, \gamma_{i}, \beta_{j}: X_{0} \times X_{0} \rightarrow \mathbb{R}_{+} \backslash\{0\}$. The problem (FI) is said to be a convex-concave fractional problem if $p_{i}$ is convex, $q_{i}$ is concave, and $g$ is convex.

THEOREM 4.1. Consider the V-invex fractional programming problem (FI). Let $a \in \Delta$. Assume that there exist $(\tau, \lambda)$ such that $\tau \geq 0, \tau \neq 0, \lambda \geq 0$, 
$\sum_{i=1}^{r} \tau_{i}\left(p_{i} / q_{j}\right)^{\prime}(a)+\sum_{j=1}^{m} \lambda_{j} g_{j}^{\prime}(a)=0$ and $\lambda_{j} g_{j}(a)=0, j=1,2, \ldots, m$. Then, $a$ is a global weak minimum for (FI).

Proof. The conclusion will follow from Theorem 3.1 if we show that there exists $\alpha_{i}: X_{0} \times X_{0} \rightarrow \mathbb{R}_{+} \backslash\{0\}$ such that

$$
x \in \Delta \Rightarrow\left\{\begin{aligned}
\left(p_{i} / q_{i}\right)(x)-\left(p_{i} / q_{i}\right)(a) & \geq \alpha_{i}(x, a)\left(p_{i} / q_{i}\right)^{\prime}(a) \eta(x, a) \\
g_{j}(x)-g_{j}(a) & \geq \beta_{j}(x, a) g_{j}^{\prime}(a) \eta(x, a) .
\end{aligned}\right.
$$

To see this, let $x \in \Delta$. Consider

$$
\begin{aligned}
p_{i}(x) q_{i}(a)-p_{i}(a) q_{i}(x) & =q_{i}(a)\left[p_{i}(x)-p_{i}(a)\right]-p_{i}(a)\left[q_{i}(x)-q_{i}(a)\right] \\
& \geq q_{i}(a) \gamma_{i}(x, a) p_{i}^{\prime}(a) \eta(x, a)-p_{i}(a) \gamma_{i}(x, a) q_{i}^{\prime}(a) \eta(x, a) \\
& =\gamma_{i}(x, a)\left[q_{i}(a) p_{i}^{\prime}(a)-p_{i}(a) q_{i}^{\prime}(a)\right] \eta(x, a),
\end{aligned}
$$

thus,

$$
\begin{aligned}
p_{i}(x) q_{i}(a)-p_{i}(a) q_{i}(x) & \geq \gamma_{i}(x, a)\left[q_{i}(a) p_{i}^{\prime}(a)-p_{i}(a) q_{i}^{\prime}(a)\right] \eta(x, a) \\
& =\gamma_{i}(x, a)\left[q_{i}(a)\right]^{2}\left(p_{i} / q_{i}\right)^{\prime}(a) \eta(x, a) .
\end{aligned}
$$

Now, dividing the inequality by $q_{i}(x) q_{i}(a)>0$, we get

$$
\frac{p_{i}(x)}{q_{i}(x)}-\frac{p_{i}(a)}{q_{i}(a)} \geq \gamma_{i}(x, a)\left[\frac{q_{i}(a)}{q_{i}(x)}\right]\left(\frac{p_{i}}{q_{i}}\right)^{\prime}(a) \eta(x, a) .
$$

Defining $\alpha_{i}: X_{0} \times X_{0} \rightarrow \mathbb{R}_{+} \backslash\{0\}$ by

$$
\alpha_{i}(x, y)= \begin{cases}\gamma_{i}(x, y) q_{i}(y) / q_{i}(x), & x, y \in \Delta \\ 1, & x \notin \Delta \text { or } y \notin \Delta,\end{cases}
$$

we get for each $x \in \Delta$

$$
\frac{p_{i}(x)}{q_{i}(x)}-\frac{p_{i}(a)}{q_{i}(a)} \geq \alpha_{i}(x, a)\left(\frac{p_{i}}{q_{i}}\right)^{\prime}(a) \eta(x, a) .
$$

It is worth noting that a convex-concave fractional problem is not an invex problem, but it is a V-invex fractional programming problem with $\eta(x, a)=$ $(x-a)$, and so our sufficiency theorem holds for the problem. For a V-invex multi-objective fractional programming problem (FI), the weak and strong duality properties hold with the following dual problem

$$
\begin{aligned}
& \text { V-Maximise }\left(p_{1}(\xi) / q_{1}(\xi), \ldots, p_{r}(\xi) / q_{r}(\xi)\right) \\
& \text { subject to } \sum_{i=1}^{r} \tau_{i}\left(p_{i} / q_{i}\right)^{\prime}(\xi)+\sum_{j=1}^{m} \lambda_{j} g_{j}^{\prime}(\xi)=0, \lambda_{j} \geq 0, \\
& \tau \geq 0, \tau e=1, \lambda_{j} g_{j}(\xi) \geq 0, j=1, \ldots, m .
\end{aligned}
$$


We remark that V-invexity ideas can be used to establish duality theorems with various other fractional programming dual problems (see [12]). Here, we have stated sufficiency and duality results in the previous section.

\section{Conclusions}

We have shown that $\mathrm{V}$-invex problems have the Lagrangian sufficiency, weak duality, and global optimality properties, and that they cover many classes of nonconvex problems which include certain nonconvex composite problems and nonlinear fractional programming problems. It can also be seen that the $\mathrm{V}$-invexity condition allows one to treat problems with certain invex functions such as strongly pseudo-convex functions and linear or affine functions that cannot be handled by the usual invexity conditions (see examples in Section 2). It is also worth pointing out that the methods of proof presented here extend to nonsmooth problems and various other kinds of fractional programming problems with appropriate modifications. Duality results for multi-objective fractional programming problems with properly efficient points and efficient points will be discussed in a forthcoming paper. This is dedicated to Bruce Craven on his 60th birthday.

\section{Acknowledgement}

The authors are indebted to Professor Pham Sach for his helpful comments on the preliminary version of the paper. This paper is dedicated to Bruce Craven on his 60th birthday.

\section{References}

[1] A. Ben-Israel and B. Mond, "What is invexity?", J. Austral. Math. Soc. Ser. B 28 (1986) 1-9.

[2] S. Chandra, "Strong pseudo-convex programming", Indian J. Pure Appl. Math. 3 (1972) 278-282.

[3] K. L. Chew and E. U. Choo, "Pseudolinearity and efficiency", Mathematical Programming 28 (1984) 226-239.

[4] B. D. Craven, "Invex functions and constrained local minima", Bull. Austral. Math. Soc. 24 (1981) 357-366.

[5] _ _ "Duality for generalised convex fractional programs", in Generalised concavity in Optimisation and Economics (eds. S. Schaible and W. T. Ziemba) (Academic Press, New York, 1981) 473-490.

[6] __, Fractional programming, (Heldermann Verlag, Berlin, 1988).

[7] _, Mathematical programming and control theory, (Chapman and Hall, London, 1978). 
[8] B. D. Craven and B. M. Glover, "Invex functions and duality", J. Austral. Math. Soc. Ser. A 39 (1985) 1-20.

[9] M. A. Hanson, “On sufficiency of the Kuhn-Tucker conditions", J. Math. Anal. Appl. 80 (1981) 544-550.

[10] D. H. Martin, "The essence of invexity", J. Optim. Theore. Appl. 47 (1985) 65-76.

[11] B. Mond, "Generalised convexity in mathematical programming", Bull. Austral. Math. Soc. 27 (1983) 185-202.

[12] B. Mond and T. Weir, "Duality for fractional programming with generalised convexity conditions", J. Inform. Optim. 3 (1982) 105-124.

[13] T. Weir, B. Mond and B. D. Craven, "On duality for weakly minimized vector valued optimisation problems", Optimisation 17 (1986) 711-721. 